\title{
EFFECTS OF NICOTINE ABSTINENCE AND MENSTRUAL PHASE ON TASK PERFORMANCE
}

\author{
CYNTHIA S. POMERLEAU, FRANÇOISE TEUSCHER, SUSAN GOETERS, \\ and OVIDE F. POMERLEAU
}

Behavioral Medicine Program, Department of Psychiatry, University of Michigan School of Medicine

\begin{abstract}
Both menstrual phase and nicotine have been shown to affect task performance. Though conflicting results have been reported, at least one well-controlled study has demonstrated that women at midluteal phase show superior performance on speech articulation and speeded motor coordination tests, but poorer performance on perceptual-spatial tests, than during menses. Smokers have demonstrated superior performance on numerous tasks following nicotine than following placebo. To explore the separate and combined influence of these factors, we studied 13 regularly-menstruating smokers using a two (smoking vs. 12 hours' abstinence) by two (menstrual vs. midluteal phase) factorial design. During each session, subjects completed a test battery including two speeded motor coordination tasks, a computerized reaction time test, and the Stroop (1935) color/word test. Subjects completed the Stroop color and color-word tasks significantly faster after ad lib smoking than after overnight abstinence. No other significant differences emerged. Our findings replicate, in an all-female sample, previous reports that speed of cognitive processing is reduced by nicotine abstinence (or enhanced by nicotine administration). Our failure to observe menstrual cycle effects raises the possibility that the anti-estrogenic effects of smoking may attenuate phase differences in performance.
\end{abstract}

Both menstrual phase and nicotine exposure have been shown to affect task performance. Although investigations of the effects of menstrual phase have produced conflicting results (see Sommer, 1983), a compelling study by Hampson and Kimura (1988) found that during the midluteal phase (when both estrogen and progesterone levels are high), women showed superior performance on speech articulation, manual dexterity, and verbal fluency (tasks in which females typically excel; Maccoby \& Jacklin, 1974) and poorer performance on maps, mazes, and perceptual-spatial tasks (tasks in which males typically excel; Witkin, Dyk, Faterson, Goodenough, \& Karp, 1962) than during menses (when both estrogen and progesterone levels are low). Recent investigations by Hampson (1990a, 1990b) and others (Gordon \& Lee, 1993) have not consistently replicated these effects; nor has any clear pattern relating performance to steroid or gonadotrophic hormone levels been demonstrated. Thus, though the findings of Hampson and Kimura (1988) are suggestive, they must still be regarded as tentative. With respect to smoking, a fairly extensive literature has been accumulated showing increases in several kinds of task performance (involving, variously, nonsmokers, deprived smokers, and nondeprived smokers) following nicotine administration as compared with placebo administration, including motor performance (e.g., finger tapping), reaction time, and focused attention (e.g., the Stroop test; Stroop, 1935) tasks (see Warburton, 1990 for a review).

Support for this research was provided by National Cancer Institute grant CA 42730 to the fourth author. The authors wish to thank Whitehall Laboratories for their generous contribution of Clearplan Easy ovulation predictor kits; Bruno Giordani, $\mathrm{PhD}$, for helpful comments on an earlier draft of this paper; and Emily Ehrlich, BA, for assistance in the conduct of this research.

Requests for reprints should be sent to Cynthia S. Pomerleau, Behavioral Medicine Program, University of Michigan Department of Psychiatry, 475 Market Place, Suite L, Ann Arbor, MI 48108. 
The current study was designed to replicate these effects in women smokers and to detcrmine whether there were interactions between the two factors. A battery of four tests was administered to 13 regularly menstruating women smokers on the days of the month specified by Hampson and Kimura (1988), in two consecutive months, with an ad lib smoking condition and an overnight abstinence condition prevailing once during each of the two menstrual phases. The tasks were chosen with the expectation that they would be sensitive to both nicotine condition and menstrual phase. Since the tasks in our test battery all fell within the category in which women excelled during the midluteal phase, we hypothesized that subjects would perform better (a) during the midluteal phase than during menses, and (b) during ad lib smoking than during nicotine abstinence.

\section{E T H O D}

\section{Subjects}

Subjects were 13 female smokers recruited via newspaper advertisements and meeting the following inclusion/exclusion criteria: age 20-39 years; regularly menstruating (i.e., cycle length predictable in the last few months, plus or minus a few days); not using oral contraceptives for a minimum of 2 months prior to the start of the study; no regular use of medications; smoked at least 2 years; cigarettes/day $\geq$ 15 , of a brand having a nicotine yield of $\geq 0.5 \mathrm{mg}$; caffeine intake $\leq 600 \mathrm{mg} /$ day. Subjects were not screened for premenstrual symptomatology; all but two responded positively to a query regarding mood changes related to menstrual phase, but the topic was not pursued further.

\section{Scheduling of sessions}

Experimental sessions were scheduled on two consecutive months - two sessions on the 3rd-5th day after the onset of menses and two sessions on the 7th-9th day after the preovulatory LH surge (i.e., during the midluteal phase). The LH surge was detected by a home urine testing kit (Clearplan Easy; Whitehall Laboratories, New York). Subjects were randomly assigned to one of eight possible orders; once started, each subject returned for a session with each subsequent midluteal or menstrual phase. By the time she completed the protocol, each subject had participated in one menstrual/ad lib smoking session, one menstrual/abstinence session, one midluteal/ad lib smoking session, and one midluteal/abstinence session.

\section{Confirmation of menstrual phase and nicotine abstinence}

Confirmation that a cycle was ovulatory was obtained via the ProgestURINE test kit (Monoclonal Antibodies, Inc., Mountain View, CA), a 5-minute enzyme immunoassay for the qualitative detection of a major urinary metabolite of progesterone, pregnanediol glucuronide, for the three mornings preceding the two midluteal sessions. At least one positive urine sample was required. Previous positive LH testing and the timing of the start of the next period ( $14 \pm 3$ days post positive $\mathrm{LH}$ ) provided additional confirmation. For the two nicotine abstinence sessions, abstinence was confirmed by expired air $\mathrm{CO}<15 \mathrm{ppm}$.

\section{Apparatus and test battery}

During experimental sessions, the subject was seated in an easy chair in a room equipped with a one-way mirror. All experimental sequences were controlled by an 
IBM AT computer. An AMIGA computer, situated adjacent to the easy chair, was used to issue instructions to the subject via a voice synthesizer, to which the subject responded using a mouse. Heart rate was monitored using a Grass model 7B polygraph (Grass Medical Instruments, Quincy, MA) and digitized using the IBM AT.

A test battery of four tasks was administered in a uniform order at each session: (a) Finger tapping: The subject was instructed to use the index finger of her dominant hand to tap the mouse button "as fast as possible." The IBM AT recorded finger taps/min for $1 \mathrm{~min}$. (b) "Reaction boxes": Ten boxes were randomly presented on the AMIGA computer screen, one after the other. The subject was instructed to use the mouse to move the cursor and click onto the box. The total number of "hits" and the total time to complete those "hits"' were recorded. (c) Stroop test (Stroop, 1935): This test was administered in accordance with the Stroop Neuropsychological Screening Test Manual (Trennery, Crossen, DeBoe, \& Leber, 1989). The subject was instructed first to read a scrambled word-list of the names of four different colors (blue, green, red, tan) printed in nonmatching colored ink (the color task), and then to read a similar word-list but to name the color of ink used for the word rather than the word itself (the color-word task). The total number of correct responses, the total number of corrected responses, the total number of incorrect responses, and the time to completion up to the maximum time allotment of $2 \mathrm{~min}$, were recorded; (4) "Pin test' (Satz \& D'Elia, 1989): The subject was trained to push a pin through a patterned set of holes so as to make indentations on a scoring sheet. The test consisted of four 30-s trials, two with each hand, beginning with the dominant hand and then switching to the nondominant hand, and alternating after each trial. The total number of holes punched for the dominant versus the nondominant hand was recorded. During the first two tasks, the experimenter sat in a contiguous room, observing the subject through a one-way mirror. During the last two tasks, the experimenter was in the room with the subject.

\section{Procedure}

Each subject participated in a screening and familiarization session in which the study was explained, informed consent was obtained, instruction was provided in the hormonal assay procedures, and a trial run of the test battery was carried out to minimize practice effects during the experimental sessions. The subject then completed a general medical, demographic, and smoking history questionnaire and the Fagerström Tolerance Questionnaire (FTQ; an 8-item assessment of degree of dependence, with possible scores ranging from 0 to 11; Fagerström, 1978) and asked to call at the appropriate time (depending on the counterbalancing sequence to which she was randomly assigned) so that experimental sessions could be scheduled.

Sessions were half an hour in length and occurred between 11:30 a.m. and 1:30 p.m., standardized within an hour for each subject. If a session was missed for any reason, the study was resumed at the same point during the subsequent cycle. During each session, the subject completed (a) The Bipolar Nicotine Withdrawal Scale (BNWS; Tate, Pomerleau, \& Pomerleau, 1993), a modification of the Hughes/ Hatsukami Smoking Withdrawal Scale (Hughes \& Hatsukami, 1986), with instructions to report how she was feeling "right now"; and (b) the test battery of four tasks as described above. During the ad lib smoking sessions, the subject was instructed to smoke one of her usual brand cigarettes $5 \mathrm{~min}$ before the administration of the withdrawal scale and the test battery. Prior to the nicotine abstinence sessions, 


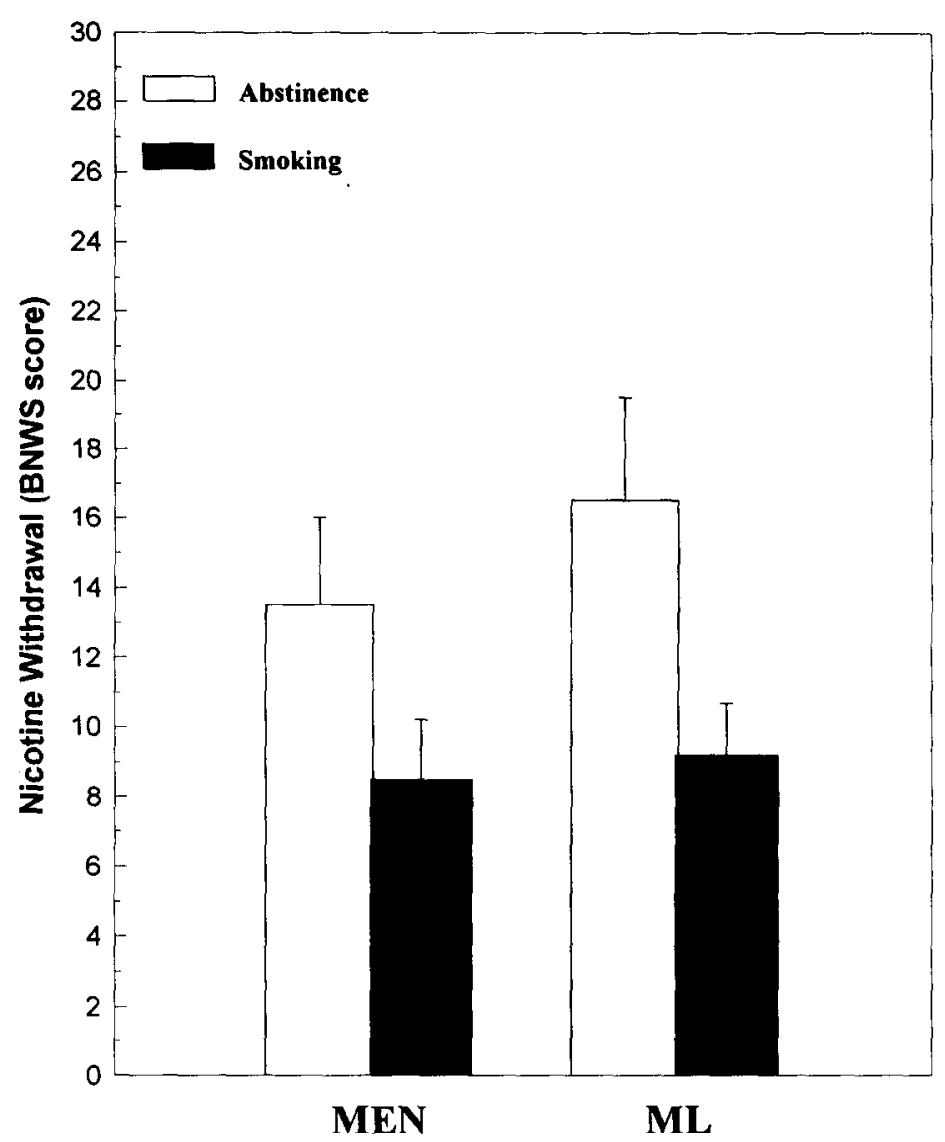

Fig. 1. Nicotine withdrawal symptomatology $(n=13)$ as measured by the Bipolar Nicotine Withdrawal Scale (Tate, Pomerleau, \& Pomerleau, 1993) under conditions of abstinence and smoking, during menses (MEN) and the midluteal (MF) phase (mean \pm SEM).

subjects had been instructed not to smoke from midnight the night before the session until after the completion of the withdrawal scale and the test battery.

\section{Data analysis}

The principal data analyses were carried out using repeated measures ANOVAs for a two (ad lib smoking vs. abstinence) by two (menstrual vs. midluteal phase) factorial design. Two-tailed tests of significance were used throughout.

\section{R E S U L T S}

Subjects had a mean age of $27.9 \pm 1.5$ SEM years. They smoked a mean of $20.2 \pm$ .9 cigarettes per day and posted a mean score of $5.9 \pm .4$ on the FTQ, characterizing them as moderately dependent smokers. Average menstrual cycle length was $28.7 \pm$ .6 days.

Expired $\mathrm{CO}$ on the abstinence days ranged from $2-13 \mathrm{ppm}$ (means $=5.8 \pm .7$ and $6.1 \pm 1.0$ for the two abstinence days), indicating good compliance with the instruction to remain abstinent for $12 \mathrm{~h}$. Mean baseline heart rate for the 2 smoking days $(5$ min after smoking a cigarette, in the context of ad lib smoking; $90.8 \pm 2.5 \mathrm{bpm}$ ) was 


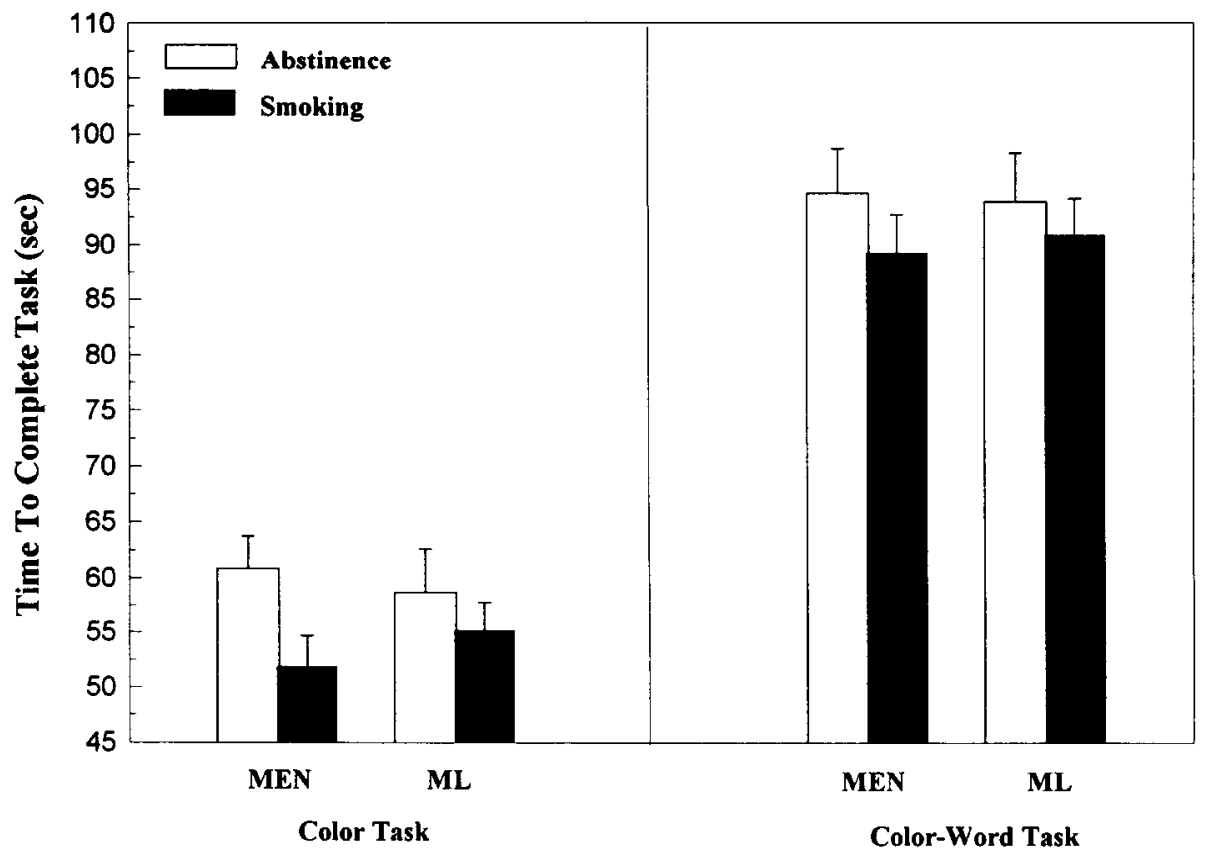

Fig. 2. Time required to complete Stroop color $(n=10)$ and color-word $(n=11)$ tasks under conditions of abstinence and smoking, during menses (MEN) and the midluteal (ML) phase (mean \pm SEM).

significantly higher than for the two abstinence days $(71.7 \pm 2.2 \mathrm{bpm} ; F[1,12]=$ $43.91, p<.0001$ ), also reflecting the effects of differential nicotine exposure. As expected, ratings on the BNWS were significantly higher for the nicotine abstinence sessions than for the ad lib smoking sessions (see Fig. $1 ; F[1,12]=6.41, p<.05$ ); no significant effect for menstrual phase was detected, nor did an interaction of phase with abstinence emerge.

As shown in Figure 2, subjects completed both the Stroop color task and the Stroop color-word task significantly faster in the context of ad lib smoking than following 12 hours' abstinence (color task [missing data for 3 subjects]: $F[1,9]=$ $9.30, p<.05$ ); color-word task [ 2 subjects excluded because they failed to complete the task within the 120 -sec limit on $\geq 2$ trials]: $F[1,10]=5.69, p<.05$ ). No other significant main or interaction effects were detected for any of the tasks.

\section{I S C U S S I O N}

Subjects completed both the Stroop color and color-word tasks in significantly less time under conditions of ad lib smoking than following 12 hours' abstinence. The finding that speed of cognitive processing may be reduced by nicotine abstinence (or, alternatively, enhanced by nicotine administration) replicates, in an all-female sample, similar findings on "Stroop interference" by other investigators (Provost \& Woodward, 1991; Wesnes \& Warburton, 1978).

Contrary to our predictions, our subjects failed to show any menstrual cycle phase effects. These findings raise the possibility that the anti-estrogenic effects of smoking (Michnovicz, Hershcopf, Naganuma, Bradlow, \& Fishman, 1986) may contribute to an attenuation of phase differences in performance, particularly performance of 
tasks such as those included in our test battery, in which peak performance was reported under conditions of higher estrogen levels. In the absence of nonsmoking controls, however, it cannot be determined whether our negative results are specific to smokers; in view of conflicting findings in the literature, the existence of menstrual phase differences in performance remains to be confirmed. It is also possible that the tests used in this study were not sufficiently sensitive to detect the expected effects. Although most of our subjects reported experiencing some degree of mood change related to menstrual phase, it is conceivable that subjects with more pronounced menstrual or premenstrual symptomatology would have been more "reactive" to phase differences, or that phase differences in performance might have been observed had subjects been tested during the premenstruum, the time at which mood changes are most likely to occur. Further research involving larger $N$ s, nonsmoking controls, perceptual-spatial as well as verbal articulation and speeded motor coordination tasks, additional menstrual phases, and assessment (or preferably manipulation) of estrogen levels will bc required beforc the possibility that nicotine and menstrual phase produce interactive effects task performance in women smokers can be ruled out altogether.

\section{R E F E R E N C E S}

Fagerström, K. O. (1978). Measuring degree of physical dependence to tobacco smoking with reference to individualization of treatment. Addictive Behaviors, 6, 345-353.

Gordon, H. W., \& Lee, P. A. (1993). No difference in cognitive performance between phases of the menstrual cycle. Psychoneuroendocrinology, 18, 521-531.

Hampson, E. (1990a). Variations in sex-related cognitive abilities across the menstrual cycle. Brain and Cognition, 14, 26-43.

Hampson, E. (1990b). Estrogen-related variations in human spatial and articulatory-motor skills. Psychoneuroendocrinology, 15, 97-111.

Hampson, E., \& Kimura, D. (1988). Reciprocal effects of hormonal fluctuations on human motor and perceptual-spatial skills. Behavioral Neuroscience, 102, 456-459.

Hughes, J. R., \& Hatsukami, D. K. (1986). Signs and symptoms of tobacco withdrawal. Archives of General Psychiatry, 32, 289-294.

Maccoby, E. E., \& Jacklin, C. N. (1974). The psychology of sex differences. Stanford, CA: Stanford University Press.

Michnovicz, J. J., Hershcopf, R. J., Naganuma, H. D., Bradlow, H. L., \& Fishman, J. (1986). Increased 2-hydroxylation of estradiol as a possible mechanism for the anti-estrogenic effect of cigarette smoking. New England Journal of Medicine, 21, 1305-1309.

Provost, S. C., \& Woodward, R. (1991). Effects of nicotine gum on repeated administration of the Stroop test. Psychopharmacology, 104, 536-540.

Satz, P., \& D'Elia, L. (1989). The pin test. Odessa, FL: Psychological Assessment Resources, Inc.

Sommer, B. (1983). How does menstruation affect cognitive competence and psychophysiological response? Women and Health, 8, 53-90.

Stroop, J. R. (1935). Studies of interference in serial verbal reactions. Journal of Experimental Psychology, 18, 643-662.

Tate, J. C., Pomerleau, O. F., \& Pomerleau, C. S. (1993). Temporal stability and within-subject consistency of nicotine withdrawal symptoms. Journal of Substance Abuse, 5, 355-363.

Trennery, M. R., Crossen, B., DeBoe, J., \& Leber, W. R. (1989). Stroop neuropsychological screening test manual. Odessa, FL: Psychological Assessment Resources, Inc.

Warburton, D. M. (1990). Psychopharmacological aspects of nicotine. In S. Wonnacott, M. A. H. Russell, \& I. P. Stolerman, (Eds), Nicotine psychopharmacology: Molecular cellular and behavioural aspects (pp. 77-111). Oxford: Oxford University Press.

Wesnes, K., \& Warburton, D. M. (1978). The effect of cigarette smoking and nicotine tablets on human attention. In R. E. Thornton (Ed), Smoking behaviour: Physiological and psychological influences. (pp. 131-147) Edinburgh: Churchill-Livingstone.

Witkin, H. A., Dyk, R. B., Faterson, H. F., Goodenough, D. R., \& Karp, S. A. (1962). Psychological differentiation. New York: Wiley. 